\title{
Left heart decompression in patients supported with extracorporeal membrane oxygenation for cardiac disease
}

\author{
Chin Siang Ong, Narutoshi Hibino \\ Division of Cardiac Surgery, Johns Hopkins Hospital, Baltimore, MD, USA
}

Adv Interv Cardiol 2017; 13, 1 (47): 1-2

DOI: https://doi.org/10.5114/aic.2017.66179

Patients on extracorporeal membrane oxygenation (ECMO) for cardiac disease frequently have some degree of left ventricular (LV) distension due to incomplete unloading. However, a chronically or excessively volume overloaded and distended LV, with persistently elevated left ventricular end-diastolic pressure (LVEDP) and elevated wall stress, impairs myocardial recovery and the ability to wean off ECMO. The presence of concomitant cardiogenic pulmonary edema, in many cases, also leads to poor patient outcomes. Thus, in order to unload and decompress the left heart, conservative, pharmacologic, interventional and surgical strategies have been described $[1,2]$.

In this issue, the authors present the use of transseptal balloon atrial septostomy (BAS) and balloon dilation, to vent the left heart, in an adult patient with dilated cardiomyopathy and severe biventricular failure on ECMO, with worsening pulmonary edema. While treatment strategies have been reviewed, the timing, indications and algorithm for left heart decompression for patients on ECMO, especially by percutaneous BAS, remain debatable. Actual treatment strategies remain customized and decided on a case-by-case basis.

With regards to the timing for left heart decompression for patients on ECMO, in one single-center case series (23 pediatric patients), it appears that earlier LA decompression (by all modalities) is associated with better LV recovery and improved likelihood of weaning from ECMO [3]. In another single-center case series (37 pediatric patients), trans-catheter procedures to decompress the left heart were performed in $97 \%$ of patients within $24 \mathrm{~h}$ of ECMO initiation [4]. In a multi-center case series (64 patients: 32 adult, 32 pediatric) and the only study to include adult patients with available data on timing of intervention, Baruteau et al. [5] report that the mean ECMO-BAS timing was 1.5 days, with a range of 0 to 12 days. In a literature summary by the same authors, pre- viously reported mean ECMO-BAS timings for pediatric patients ranged from $8 \mathrm{~h}$ to 2.5 days.

A review of the literature found the following indications for left heart decompression [1-10]:

1. Hearts with no obvious ejection and a closed aortic valve (as in the patient discussed in this issue),

2. Refractory pulmonary edema (as in the patient discussed in this issue),

3. Distended left atrium (LA) and LV with elevated LA pressure and LVEDP, despite maximal pharmacologic support,

4. Pulmonary hemorrhage,

5. Significant aortic valve regurgitation,

6. LV thrombus secondary to stasis,

7. Impaired RV function,

8. Insufficient LV unloading by ECMO circuit,

9. Elevated LV wall stress (decompression to reduce LV wall stress),

10. Severe or persistent LV dysfunction (decompression to aid in LV recovery).

Interventional approaches to decompress the left heart include cardiac support interventions, such as intra-aortic balloon pumps, and percutaneous techniques to vent the left heart, such as pulmonary artery vents, retrograde transaortic vents, transseptal LA decompression [6,9] with BAS and LA vent insertion [9], static overthe-wire [5, 9] balloon dilation, blade balloon septostomy $[7,9]$ and atrial stenting $[9,10]$. The combination of BAS with ECMO has been shown to be safe and efficient in 64 patients in a multi-institutional study [5]. For patients with severe left ventricular failure with a competent mitral valve, the role of BAS is limited to reduction of pulmonary edema. If BAS is not effective to decompress the left ventricle, an early decision of switching from ECMO to a left ventricular assist device would be warranted.

In conclusion, the decision to decompress the left heart in patients on ECMO is a multifactorial one. Evi-

\section{Corresponding author:}

Narutoshi Hibino MD, PhD, Assist. Prof., Division of Cardiac Surgery, The Johns Hopkins Hospital, Sheikh Zayed Tower - Suite 7107, 1800 Orleans St, Baltimore, MD 21287, USA, e-mail: nhibino1@jhmi.edu

Received: 14.02.2017, accepted: 14.02.2017. 
dence-based guidelines, treatment algorithms and consensus statements will be beneficial to guide management. An early decision of interventional approaches such as BAS would be beneficial for some patients, but switching to a left ventricular assist device is needed for most patients with severe left ventricular failure.

\section{Conflict of interest}

The authors declare no conflict of interest.

\section{References}

1. Rupprecht L, Florchinger B, Schopka S, et al. Cardiac decompression on extracorporeal life support: a review and discussion of the literature. ASAIO J 2013; 59: 547-53.

2. Gultekin B, Ersoy O, Akkaya I, et al. Decompression of left ventricle during venoarterial extracorporeal membrane oxygenation support as a step to transplant. Exp Clin Transplant 2016; 14: 42-4.

3. Kotani Y, Chetan D, Rodrigues W, et al. Left atrial decompression during venoarterial extracorporeal membrane oxygenation for left ventricular failure in children: current strategy and clinical outcomes. Artificial Organs 2013; 37: 29-36.

4. O’Byrne ML, Glatz AC, Rossano JW, et al. Middle-term results of transcatheter creation of atrial communication in patients receiving mechanical circulatory support. Catheter Cardiovasc Interv 2015; 85: 1189-95.

5. Baruteau AE, Barnetche T, Morin L, et al. Percutaneous balloon atrial septostomy on top of venoarterial extracorporeal membrane oxygenation results in safe and effective left heart decompression. Eur Heart J Acute Cardiovasc Care 2016; in press.

6. Aiyagari RM, Rocchini AP, Remenapp RT, Graziano JN. Decompression of the left atrium during extracorporeal membrane oxygenation using a transseptal cannula incorporated into the circuit. Crit Care Med 2006; 34: 2603-6.

7. Johnston TA, Jaggers J, McGovern JJ, O'Laughlin MP. Bedside transseptal balloon dilation atrial septostomy for decompression of the left heart during extracorporeal membrane oxygenation. Catheter Cardiovasc Interv 1999; 46: 197-9.

8. Eudailey KW, Yi SY, Mongero LB, et al. Trans-diaphragmatic left ventricular venting during peripheral venous-arterial extracorporeal membrane oxygenation. Perfusion 2015; 30: 701-3.

9. Eastaugh LJ, Thiagarajan RR, Darst JR, et al. Percutaneous left atrial decompression in patients supported with extracorporeal membrane oxygenation for cardiac disease. Pediatr Crit Care Med 2015; 16: 59-65.

10. Haynes S, Kerber RE, Johnson FL, et al. Left heart decompression by atrial stenting during extracorporeal membrane oxygenation. Int J Artif Organs 2009; 32: 240-2. 